\title{
Boundary Non-crossings of Brownian Pillow
}

\author{
Enkelejd Hashorva
}

Received: 6 April 2008 / Revised: 6 September 2008 / Published online: 30 October 2008

(C) Springer Science+Business Media, LLC 2008

Abstract Let $B_{0}(s, t)$ be a Brownian pillow with continuous sample paths, and let $h, u:[0,1]^{2} \rightarrow \mathbb{R}$ be two measurable functions. In this paper we derive upper and lower bounds for the boundary non-crossing probability

$$
\psi(u ; h):=\mathbf{P}\left\{B_{0}(s, t)+h(s, t) \leq u(s, t), \forall s, t \in[0,1]\right\} .
$$

Further we investigate the asymptotic behaviour of $\psi(u ; \gamma h)$ with $\gamma$ tending to $\infty$ and solve a related minimisation problem.

Keywords Boundary non-crossing probability $\cdot$ Brownian pillow with trend $\cdot$ Large deviations · Smallest concave majorant · Reproducing kernel Hilbert space $\cdot$ Small ball probabilities

Mathematics Subject Classification (2000) Primary 60J65 - Secondary 60F10 * 60G15 - 60G70

\section{Introduction}

Let $B_{0}(s, t), s, t \in[0,1]$ be a Brownian pillow with continuous sample paths. Its covariance function $K$ is the product of two covariance functions defined by

$$
K\left(\left(s_{1}, t_{1}\right),\left(s_{2}, t_{2}\right)\right)=K_{1}\left(s_{1}, t_{1}\right) K_{2}\left(s_{2}, t_{2}\right), \quad s_{i}, t_{i} \in[0,1], i=1,2,
$$

with $K_{i}(s, t)=\min (s, t)-t s, i=1,2$, the covariance function of a Brownian bridge.

E. Hashorva $(\bowtie)$

Department of Mathematical Statistics and Actuarial Science, University of Bern, Sidlerstrasse 5,

3012 Bern, Switzerland

e-mail: enkelejd.hashorva@stat.unibe.ch 
Our concern in this article is the boundary non-crossing probability

$$
\psi(u ; h):=\mathbf{P}\left\{B_{0}(s, t)+h(s, t) \leq u(s, t), \forall s, t \in[0,1]\right\}
$$

with a trend function $h$ and a measurable boundary function $u$.

When considering a Brownian bridge and a Brownian motion, the corresponding non-crossing probability can be explicitly calculated if $h$ and $u$ are polygonal lines, see e.g. $[5,11,14,26,29]$ and the references therein. Such explicit formulae are not available in our setup of the multi-parameter processes.

Our novel results presented below are:

(a) upper and lower bounds for $\psi(u ; h)$,

(b) a large deviation type result for the boundary non-crossing probability $\psi(u ; \gamma h)$

with $\gamma \rightarrow \infty$, and

(c) we solve a related minimisation problem.

We comment briefly the result mentioned in (b). Given a function $g:[0, \infty)^{2} \rightarrow \mathbb{R}$, we denote by $g^{\prime \prime}$ its partial derivative obtained by differentiating both components, provided that it exists. From the large deviation theory (see e.g. [24] or [21]) for any positive constant $c$ and any trend function $h:[0,1]^{2} \rightarrow \mathbb{R}$ with a square-integrable partial derivative $h^{\prime \prime}$ (i.e. $\left.\int_{[0,1]^{2}}\left(h^{\prime \prime}(s, t)\right)^{2} d s d t<\infty\right)$, we obtain

$$
\begin{gathered}
\lim _{\gamma \rightarrow \infty} 2 \gamma^{-2} \ln \boldsymbol{P}\left\{\sup _{s, t \in[0,1]}\left(B_{0}(s, t)+\gamma h(s, t)\right) \leq c\right\} \\
=-\int_{[0,1]^{2}}\left(\underline{h}^{\prime \prime}(s, t)\right)^{2} d s d t \in(-\infty, 0]
\end{gathered}
$$

with $\underline{h}$ the solution of the minimisation problem

$$
\inf _{g \geq h} \int_{[0,1]^{2}}\left(g^{\prime \prime}(s, t)\right)^{2} d s d t,
$$

where the functions $g:[0,1]^{2} \rightarrow \mathbb{R}$ in the minimisation problem are assumed to possess a square-integrable partial derivative $g^{\prime \prime}$, and $g, h$ vanish on the boundary of $[0,1]^{2}$.

Compared to (1.2), our new result is a sharper asymptotic estimate of the boundary non-crossing probability of interest. In the special case $h$ being a product of two concave functions $h_{1}, h_{2}:[0,1] \rightarrow[0, \infty)$ with $h_{i}(0)=h_{i}(1)=0, i=1,2$, we show (see below (4.5))

$$
\begin{aligned}
& \boldsymbol{P}\left\{\sup _{s, t \in[0,1]}\left(B_{0}(s, t)+\gamma h_{1}(s) h_{2}(t)\right) \leq c\right\} \\
& \quad=\exp \left(-\frac{\gamma^{2}}{2} \prod_{i=1,2} \int_{[0,1]}\left(h_{i}^{\prime}(x)\right)^{2} \lambda(d x)+c \gamma \prod_{i=1,2}\left[h_{i}^{\prime}(1)-h_{i}^{\prime}(0)\right]+z(\gamma)\right),
\end{aligned}
$$

where

$$
-A \gamma^{2 / 3} \ln ^{3} \gamma \leq z(\gamma) \leq \ln \boldsymbol{P}\left\{\sup _{s, t \in[0,1]} B_{0}(s, t) \leq c\right\}
$$


holds for all large $\gamma$ with positive constant $A$ not depending on $\gamma$. Here $h_{i}^{\prime}$ is a rightcontinuous version of the derivative of $h_{i}, i=1,2$, and $\lambda$ is the Lebesgue measure on $[0,1]$.

We derive (1.4) utilising a known small ball result for a Brownian pillow. Indeed the small ball problem for both a Brownian pillow and a Brownian sheet is investigated by several authors, see [6-8, 10, 16-18, 20, 23, 28] among many other references.

A consequence of the Gaussian shift inequality (see [22]) and (1.4) is the following bound (set $D$ for the set of all concave functions $f:[0,1] \rightarrow[0, \infty)$ ):

$$
\boldsymbol{P}\left\{\sup _{s, t \in[0,1]} B_{0}(s, t) \leq c\right\} \leq \inf _{h \in D} \Phi\left(c^{2}\left(\frac{h^{\prime}(1)-h^{\prime}(0)}{\int_{0}^{1}\left(h^{\prime}(x)\right)^{2} \lambda(d x)}\right)^{2}\right)
$$

with $\Phi$ the distribution function of a Gaussian random variable with mean 0 and variance 1 . Since the upper bound in (1.5) is not smaller than $1 / 2$, the above inequality is of some interest, provided that $\psi(0 ; c) \in(1 / 2,1)$.

Organisation of the paper: In the next section we present some notation and preliminary results. The main results are discussed in Sect. 3. Section 4 explains the simple situation where the trend function $h$ is a product of two trend functions. Proofs of all the results are relegated to Sect. 5 followed by a short Appendix with two results on the Riemann-Stieltjes integral.

\section{Preliminaries}

We first introduce a Hilbert space related to the covariance function of a Brownian pillow, which can also be seen as tensor product of Hilbert spaces related to the covariance function of a Brownian bridge. Then we provide a result utilised in solving the minimisation problem (1.3).

The reproducing kernel Hilbert space (RKHS) related to the covariance function of a Brownian pillow, denoted by $\mathcal{H}_{2}^{0}$, is given by

$$
\begin{aligned}
\mathcal{H}_{2}^{0}:=\left\{h:[0,1]^{2} \rightarrow \mathbb{R} \mid \exists h^{\prime \prime} \in L_{2}\left([0,1]^{2}, \lambda^{2}\right),\right. \text { with } \\
h(s, t)=\int_{[0, s] \times[0, t]} h^{\prime \prime}(x, y) \lambda^{2}(d x, d y), \\
h(0, s)=h(1, s)=h(t, 0)=h(t, 1)=0, \forall s, t \in[0,1]\},
\end{aligned}
$$

where $L_{2}\left([0,1]^{2}, \lambda^{2}\right)$ is the set of all real functions on $[0,1]^{2}$ square integrable with respect to the Lebesgue measure $\lambda^{2}$ on $[0,1]^{2}$. The inner product is

$$
\left\langle h_{1}, h_{2}\right\rangle=\int_{[0,1]^{2}} h_{1}^{\prime \prime}(x, y) h_{2}^{\prime \prime}(x, y) \lambda^{2}(d x, d y), \quad h_{1}, h_{2} \in \mathcal{H}_{2}^{0},
$$

and the corresponding norm of $h \in \mathcal{H}_{2}^{0}$ is $\|h\|:=\langle h, h\rangle^{1 / 2}$. 
As shown in [17], another approach to deal with $\mathcal{H}_{2}^{0}$ is to construct this Hilbert space as the tensor product of two RKHS, i.e. $\mathcal{H}_{2}^{0}=\mathcal{H}_{1}^{0} \otimes \mathcal{H}_{1}^{0}$ with the RKHS $\mathcal{H}_{1}^{0}$ of the covariance function of a Brownian bridge defined by

$$
\begin{aligned}
& \mathcal{H}_{1}^{0}:=\left\{h:[0,1] \rightarrow \mathbb{R} \mid \exists h^{\prime} \in L_{2}([0,1], \lambda)\right. \text { with } \\
&\left.\quad h(s)=\int_{[0, s]} h^{\prime}(x) \lambda(d x), h(0)=h(1)=0\right\},
\end{aligned}
$$

where $L_{2}([0,1], \lambda)$ is the set of all real functions on $[0,1]$ square integrable with respect to $\lambda$. The inner product of $\mathcal{H}_{1}^{0}$ is

$$
\left\langle h_{1}, h_{2}\right\rangle=\int_{[0,1]} h_{1}^{\prime}(x) h_{2}^{\prime}(x) \lambda(d x), \quad h_{1}, h_{2} \in \mathcal{H}_{1}^{0},
$$

and the corresponding norm is denoted again by $\|\cdot\|$. Any element $h \in \mathcal{H}_{2}^{0}$ can be identified by $h_{1}, h_{2} \in \mathcal{H}_{1}^{0}$ so that $h=h_{1} \otimes h_{2}$ (see [17]).

In the following, for any trend function $h \in \mathcal{H}_{2}^{0}$, we denote by $h^{\prime \prime}$ its rightcontinuous derivative.

Lemma 2 in [15] is crucial for our next result. Define the closed convex sets

$$
\begin{aligned}
& V:=\left\{h \in \mathcal{H}_{2}^{0}: h(s, t) \leq 0, \forall s, t \in[0,1]\right\}, \\
& W:=\left\{h \in \mathcal{H}_{2}^{0}: h(s, t) \geq 0, \forall s, t \in[0,1]\right\},
\end{aligned}
$$

and let $\widetilde{V}, \widetilde{W}$ be the polar cones of $V$ and $W$, respectively, defined by

$$
\widetilde{V}:=\left\{h \in \mathcal{H}_{2}^{0}:\langle h, v\rangle \leq 0, \forall v \in V\right\}, \quad \widetilde{W}:=\left\{h \in \mathcal{H}_{2}^{0}:\langle h, v\rangle \geq 0, \forall v \in W\right\} .
$$

Further denote by $B V_{H}(T), T \subset \mathbb{R}^{2}$ the class of functions $f: T \rightarrow \mathbb{R}$ which have bounded variation in the sense of Hardy (see e.g. [1, 25]).

Lemma 2.1 Let $h \in \mathcal{H}_{2}^{0}$ be a given function, and let $V_{p, h}, \widetilde{V}_{p, h}$ be the unique projections of $h$ into $V$ and the polar cone $\widetilde{V}$, respectively.

(a) If $\widetilde{V}_{p, h}^{\prime \prime}$ is a right-continuous partial derivative of $\widetilde{V}_{p, h}$ such that $\widetilde{V}_{p, h}^{\prime \prime} \in$ $B V_{H}\left([0,1]^{2}\right)$, then for any function $g:[0,1]^{2} \rightarrow[0, \infty)$ Riemann-Stieltjes integrable with respect to $\widetilde{V}_{p, h}^{\prime \prime}$, the Riemann-Stieltjes integral $I(g):=\int_{[0,1]^{2}} g(s, t)$ $d \widetilde{V}_{p, h}^{\prime \prime}(s, t)$ satisfies $I(g) \geq 0$.

(b) We have

$$
h=V_{p, h}+\widetilde{V}_{p, h}, \quad\left\langle V_{p, h}, \widetilde{V}_{p, h}\right\rangle=0 .
$$

(c) If $h=h_{1}+h_{2}$ with $h_{1} \in V, h_{2} \in \widetilde{V}$ such that $\left\langle h_{1}, h_{2}\right\rangle=0$, then $h_{1}=V_{p, h}$ and $h_{2}=\widetilde{V}_{p, h}$.

(d) The unique solution $\underline{h}$ of the minimisation problem

$$
\min _{g \geq h, g \in \mathcal{H}_{2}^{0}}\|g\|
$$

is $\underline{h}=\widetilde{V}_{p, h}$ satisfying further $\|\underline{h}\|=\min \{\|g\|: g \in \widetilde{V}, g \geq h\}$. 
We note in passing that a similar decomposition to (2.1) can be stated for $h \in \mathcal{H}_{2}^{0}$ in terms of the unique projections $W_{p, h}, \widetilde{W}_{p, h}$ of $h$ into $W$ and the polar cone $\widetilde{W}$, respectively. Furthermore, (b) and (c) hold for some general Hilbert space.

We write alternatively $\underline{h}, \bar{h}$ instead of $\widetilde{V}_{p, h}, \widetilde{W}_{p, h}$. The above lemma immediately implies

$$
\begin{aligned}
\bar{h}(s, t) & \leq h(s, t) \leq \underline{h}(s, t), \quad \forall s, t \in[0,1], \quad \text { and } \\
\|h\| & \geq \max (\|\underline{h}\|,\|\bar{h}\|), \quad \forall h \in \mathcal{H}_{2}^{0} .
\end{aligned}
$$

Furthermore, for any two functions $h, q \in \mathcal{H}_{2}^{0}$ such that $q \geq h,(1.3)$ and Lemma 2.1 yield

$$
\|q\| \geq\|h\|,
$$

provided that $\underline{h}=h, \underline{q}=q$.

\section{Main Results}

Let $B_{0}(s, t), s, t \in[0,1]$ be a Brownian pillow with continuous sample paths, and let $h \in \mathcal{H}_{2}^{0}$ be a given trend function. For some measurable boundary function $u$ : $[0,1]^{2} \rightarrow \mathbb{R}$, we define the boundary non-crossing probability $\psi(u ; h)$ as in (1.1). Throughout the rest of the paper we assume that $\psi(u ; 0) \in(0,1)$. Since $h \in \mathcal{H}_{2}^{0}$, the Cameron-Martin formula (see e.g. [19, 22, 24] or [23]) implies

$$
\begin{aligned}
\psi(u ; h)= & \exp \left(-\frac{1}{2}\|h\|^{2}\right) \\
& \times \mathbf{E}\left\{\exp \left(\int_{[0,1]^{2}} h^{\prime \prime}(s, t) d B_{0}(s, t)\right) \mathbf{1}\left(B_{0}(s, t) \leq u(s, t), \forall s, t \in[0,1]\right)\right\},
\end{aligned}
$$

where $\mathbf{1}(\cdot)$ is the indicator function.

Li and Kuelbs [22] show that the Cameron-Martin translation implies important shift inequalities for some general Gaussian processes. Applying their Theorem 1', we have

$$
\Phi(\theta-\|h\|) \leq \psi(u ; h) \leq \Phi(\theta+\|h\|),
$$

where $\Phi$ is the Gaussian distribution function on $\mathbb{R}$ with mean 0 and variance 1 , and $\theta$ is such that $\Phi(\theta)=\psi(u ; 0)$. When $\|h\|$ is small, the lower and upper bounds in (3.2) are close to the non-crossing probability of interest, since $\lim _{\gamma \rightarrow 0} \psi(u ; \gamma h)=$ $\psi(u ; 0)=\Phi(\theta)$. As $\gamma \rightarrow \infty$, the upper bound in (3.2) tends to 1 , whereas the lower bound and $\psi(u ; \gamma h)$ tend to 0 . Note in passing that as in [27] we obtain

$$
|\psi(u ; \gamma h)-\psi(u ; 0)| \leq 2 \Phi(\gamma\|h\| / 2)-1 \leq \frac{\gamma\|h\|}{\sqrt{2 \pi}}, \quad \forall \gamma \in(0, \infty) .
$$


One important criteria which we will look at when discussing bounds for the noncrossing probability of interest is their performance for both small or large trend functions. In our first result below we provide upper and lower bounds for the boundary non-crossing probability $\psi(u ; h)$. If we consider further the trend function $\gamma h$, then the bounds perform well as $\gamma \rightarrow 0$.

Proposition 3.1 Let $h, u:[0,1]^{2} \rightarrow \mathbb{R}$ be two measurable functions such that $\psi(u ; 0) \in(0,1)$. If $h \in \mathcal{H}_{2}^{0}$, then we have

$$
\Phi(\theta-\|\underline{h}\|) \leq \psi(u ; h) \leq \Phi(\theta+\|\bar{h}\|), \quad \theta:=\Phi^{-1}(\psi(u ; 0)),
$$

with $\underline{h}, \bar{h}$ as defined in Sect. 2 and $\Phi^{-1}$ the inverse of $\Phi$. Furthermore

$$
-\frac{\|\underline{h}\|}{\sqrt{2 \pi}} \leq \psi(u ; h)-\psi(u ; 0) \leq \frac{\|\bar{h}\|}{\sqrt{2 \pi}} .
$$

When $h \neq \underline{h}$ or $h \neq \bar{h}$, in view of (2.3), we see that (3.5) yields better bounds than (3.3). By (3.5) we obtain

$$
-\gamma \frac{\|\underline{h}\|}{\sqrt{2 \pi}} \leq \psi(u ; \gamma h)-\psi(u ; 0) \leq \gamma \frac{\|\bar{h}\|}{\sqrt{2 \pi}}, \quad \forall \gamma>0,
$$

which is of some interest as $\gamma$ tends to 0 , since both the lower and upper bounds converge to 0 .

As mentioned in the Introduction, if $\gamma$ tends to infinity, then we have the logarithmic asymptotic behaviour

$$
\lim _{\gamma \rightarrow \infty} 2 \gamma^{-2} \ln \psi(u ; \gamma h)=-\|\underline{h}\|^{2}, \quad \forall h \in \mathcal{H}_{2}^{0},
$$

with $\underline{h}$ the unique solution of the minimisation problem (2.2).

Next, we derive explicit upper and lower bounds for $\psi(u ; h)$, which perform asymptotically better (for trend function becoming large) than those implied by (3.4).

Proposition 3.2 Let $h \in \mathcal{H}_{2}^{0}$ be a given trend function, and let $u, l:[0,1]^{2} \rightarrow \mathbb{R}$ be two measurable functions. If the partial derivative $\underline{h}^{\prime \prime}$ of the projection of $h$ into its polar cone satisfies $\underline{h}^{\prime \prime} \in B V_{H}\left([0,1]^{2}\right)$ and is right continuous, then

$$
\underline{h}:=\inf _{g \geq h, g \in \widetilde{V}, g \in B V_{H}\left([0,1]^{2}\right)} g,
$$

and further $\underline{h}$ is the smallest majorant of $h$ such that its right-continuous partial derivative belongs to $B V_{H}\left([0,1]^{2}\right)$ and generates a finite positive measure.

Moreover, if the Riemann-Stieltjes integral $\int_{[0,1]^{2}} v(s, t) d \underline{h}^{\prime \prime}(s, t)$ is finite for both $v=l$ and $v=u$ and $\psi(u ; 0) \in(0,1)$, then

$$
\psi(u ; h) \leq \psi(u ; h-\underline{h}) \exp \left(-\frac{1}{2}\|\underline{h}\|^{2}+\int_{[0,1]^{2}} u(s, t) d \underline{h}^{\prime \prime}(s, t)\right)
$$


and

$$
\begin{aligned}
\psi(u ; h) \geq & \mathbf{P}\left\{l(s, t) \leq B_{0}(s, t) \leq u(s, t), \forall s, t \in[0,1]\right\} \\
& \times \exp \left(-\frac{1}{2}\|\underline{h}\|^{2}+\int_{[0,1]^{2}} l(s, t) d \underline{h}^{\prime \prime}(s, t)\right) .
\end{aligned}
$$

\section{Remarks}

(a) If $u(s, t):=c \in(0, \infty), \forall s, t \in[0,1]$, then (3.9) implies

$$
\begin{aligned}
\psi(c ; h) \leq & \psi(c ; h-\underline{h}) \\
& \times \exp \left(-\frac{1}{2}\|\underline{h}\|^{2}+c\left[\underline{h}^{\prime \prime}(1,1)-\underline{h}^{\prime \prime}(1,0)-\underline{h}^{\prime \prime}(0,1)+\underline{h}^{\prime \prime}(0,0)\right]\right) .
\end{aligned}
$$

A lower bound for $\psi(c ; h)$ is derived using (3.10) with $l(s, t):=-c$, $\forall s, t \in[0,1]$.

(b) As in the proof of Proposition 3.2, it can be shown that if the trend function $h \in \mathcal{H}_{2}^{0}$ is such that its right-continuous partial derivative $h^{\prime \prime}$ satisfies $h^{\prime \prime} \in B V_{H}\left([0,1]^{2}\right)$ and furthermore $h^{\prime \prime}$ generates a positive measure on $[0,1]^{2}$, then the unique solution of the minimisation problem (2.2) is $\underline{h}=h$.

(c) An upper bound for $\psi(u ; h)$ is the discrete boundary non-crossing probability

$$
\psi_{n}(u ; h):=\mathbf{P}\left\{B_{0}\left(s_{i}, t_{i}\right)+h\left(s_{i}, t_{i}\right) \leq u\left(s_{i}, t_{i}\right), \forall\left(s_{i}, t_{i}\right) \in T_{n}\right\}
$$

with $T_{n}:=\left\{\left(s_{i}, t_{i}\right), i=1, \ldots, n\right\} \subset[0,1]^{2}$. Hashorva [13] shows the asymptotic behaviour (considering a Brownian bridge) of the corresponding discrete boundary non-crossing probability.

Next, we discuss the asymptotic behaviour of $\psi(u ; \gamma h)$ as $\gamma \rightarrow \infty$. Exact asymptotics of the non-crossing probabilities of the Brownian motion with trend is derived in [12], which was motivated by a large deviation type result obtained in [3]. As in [4], we expect that our novel asymptotic result will have some implications for statistical applications.

Proposition 3.3 Let $h, \underline{h}, u$ be as in Proposition 3.2. Suppose that there exist functions $u_{\varepsilon} \in \mathcal{H}_{2}^{0}, \varepsilon>0$, such that $\left\|u_{\varepsilon}\right\|=O(1 / \varepsilon)$ and

$$
\lim _{\varepsilon \rightarrow 0} u_{\varepsilon}(s, t)=u(s, t), \quad u_{\varepsilon}(s, t) \leq u(s, t)-\varepsilon, \forall s, t \in[0,1] .
$$

If the Riemann-Stieltjes integral $I_{\epsilon}:=\int_{[0,1]^{2}} u_{\epsilon}(s, t) d \underline{h}^{\prime \prime}(s, t)$ exists and $\left|I_{\epsilon}\right| \leq$ $M \in(0, \infty), \forall \epsilon>0$, then

$$
\lim _{\epsilon \rightarrow 0} I_{\epsilon}=I:=\int_{[0,1]^{2}} u(s, t) d \underline{h}^{\prime \prime}(s, t), \quad|I| \leq M,
$$


and

$$
\psi(u ; \gamma h)=\exp \left(-\frac{\gamma^{2}}{2}\|\underline{h}\|^{2}+\gamma \int_{[0,1]^{2}} u(s, t) d \underline{h}^{\prime \prime}(s, t)+z(\gamma)\right),
$$

where for all large $\gamma$,

$$
\begin{aligned}
-A \gamma^{2 / 3} \ln ^{3} \gamma & \leq z(\gamma) \\
& \leq \ln \mathbf{P}\left\{B_{0}(s, t) \leq u(s, t), \forall s, t \in[0,1]: \underline{h}(s, t)=h(s, t)\right\}
\end{aligned}
$$

with positive constant A not depending on $\gamma$.

In view of the above asymptotics and (3.4), we obtain a simple upper bound for $\psi(u ; 0)$.

Corollary 3.4 Let $u:[0,1]^{2} \rightarrow \mathbb{R}$ be a measurable function satisfying the assumptions of Proposition 3.3. Then we have

$$
\psi(u ; 0) \leq \inf _{h \in \mathcal{H}_{2}^{0}, h^{\prime \prime} \in B V_{H}\left([0,1]^{2}\right):\|\underline{h}\|>0} \Phi\left(\|\underline{h}\|^{-1} \int_{[0,1]^{2}} u(s, t) d \underline{h}^{\prime \prime}(s, t)\right) .
$$

\section{Remarks}

(a) If the function $u$ in Proposition 3.3 satisfies $u(s, t)>\mu \in(0, \infty), \forall s, t \in[0,1]$, where $(s, t)$ belongs to the boundary of $[0,1]^{2}$, and there exist functions $w_{\varepsilon}$ : $[0,1]^{2} \rightarrow \mathbb{R}, \varepsilon>0$ such that $u w_{\varepsilon} \in \mathcal{H}_{2}^{0}, \varepsilon>0$, then we may define $u_{\varepsilon}$ in Proposition 3.3 by $u_{\varepsilon}:=u w_{\varepsilon}-\epsilon, \epsilon>0$. When $u$ is a positive constant, then functions $u_{\varepsilon}, \varepsilon>0$, satisfying the assumption of Proposition 3.3 can be easily constructed. If $u_{\varepsilon}$ is continuous, then the Riemann-Stieltjes integral $I_{\varepsilon}:=$ $\int_{[0,1]^{2}} u_{\varepsilon}(s, t) d \underline{h}^{\prime \prime}(s, t)$ in Proposition 3.3 is finite.

(b) When $\underline{h}^{\prime \prime}$ is almost surely continuous with respect to the Lebesgue measure $\lambda^{2}$, then instead of assuming that $\underline{h}$ has a bounded variation in the sense of Hardy (Lemma 2.1, Propositions 3.2 and 3.3) we may impose the weaker assumption that $\underline{h}$ has a bounded variation in the sense of Vitali (see Appendix below and Lemma 6.2).

(c) Our results can be easily extended to the $d$-dimensional setup by considering a Brownian pillow $B_{0}\left(s_{1}, \ldots, s_{d}\right), s_{i} \in[0,1], i \leq d$, with continuous sample paths. The term $\ln ^{3} \gamma$ in (3.15) should then be replaced by $\ln ^{2 d-1} \gamma$.

(d) Similar results can be stated for considering instead of $B_{0}$ a Brownian sheet $B(s, t), s, t \in[0, \infty)$, with continuous sample paths. For instance Proposition 3.2 holds with $\underline{h}$ the solution of the minimisation problem (1.3), where $g, h$ have square-integrable partial derivatives satisfying further $g(0, s)=h(0, s)=$ $h(t, 0)=g(t, 0)=0, s, t \in[0, \infty)$.

\section{Product Trend Functions}

As demonstrated in the previous section, the non-crossing probability $\psi(u ; h)$ can be bounded by some functions which depend on the solution of the minimisation prob- 
lem (2.2). We discuss below an instance where the solution of (2.2) can be easily determined. Let therefore $h_{1}, h_{2} \in \mathcal{H}_{1}^{0}$, and let $B_{0}(s), s \in[0,1]$, denote a Brownian bridge with continuous sample paths. If $u_{1}, u_{2}:[0,1] \rightarrow \mathbb{R}$ are two measurable functions with $u_{i}(0), u_{i}(1)>0, i=1,2$, then we have (see [2])

$$
\begin{aligned}
\mathbf{P}\left\{B_{0}(s)+h_{i}(s) \leq u_{i}(s), \forall s \in[0,1]\right\} \\
\leq \mathbf{P}\left\{B_{0}(s) \leq u_{i}(s)+\widetilde{h}_{i}(s)-h_{i}(s), \forall s \in[0,1]\right\} \\
\quad \times \exp \left(-\frac{1}{2}\left\|\widetilde{h}_{i}\right\|^{2}+\int_{[0,1]} u_{i}(s) d\left(-\widetilde{h}_{i}^{\prime}(s)\right)\right),
\end{aligned}
$$

where $\widetilde{h}_{i}, i=1,2$, is the smallest concave majorant of $h_{i}$, and $\widetilde{h}_{i}^{\prime}$ is a right-continuous derivative of $\widetilde{h}_{i}$. Furthermore, $\widetilde{h}_{i}$ is the unique solution of the minimisation problem

$$
\min _{g \in \mathcal{H}_{1}^{0}, g \geq h_{i}}\|g\|, \quad i=1,2 .
$$

Set in the following $h(s, t):=h_{1}(s) h_{2}(t), \widetilde{h}(s, t):=\widetilde{h}_{1}(s) \widetilde{h}_{2}(t), s, t \in[0,1]$, and write $h=h_{1} \times h_{2}, \tilde{h}=\tilde{h}_{1} \times \tilde{h}_{2}$. In the next lemma we show that for special trend functions, the unique solution of (2.2) with $h=h_{1} \times h_{2} \in \mathcal{H}_{2}^{0}$ is simply $\widetilde{h}$.

Lemma 4.1 Let $h:=h_{1} \times h_{2}, h_{1}, h_{2} \in \mathcal{H}_{1}^{0}$, and denote by $\widetilde{h}_{i}, i=1,2$, the smallest concave majorant of $h_{i}, i=1,2$. If

$$
\widetilde{h}(s, t) \geq h(s, t), \quad \forall s, t \in[0,1],
$$

then the unique solution $\underline{h}$ of (1.3) is $\underline{h}:=\widetilde{h}$.

Clearly, (4.2) holds if $h_{1}, h_{2}$ are both nonnegative functions. In the special case that also $u$ is a product function we have the following immediate result.

Corollary 4.2 Let $h_{i}, \widetilde{h}_{i}, i=1,2$, satisfy the assumption of Lemma 4.1, and let $u_{i}, l_{i}:[0,1] \rightarrow \mathbb{R}, i=1,2$, be measurable functions. If the Riemann-Stieltjes integral $\int_{[0,1]} v_{i}(s) d\left(-\widetilde{h}_{i}^{\prime}(s)\right)$ is a finite constant for $i=1,2$ and $v_{i}=l_{i}$ or $v_{i}=u_{i}$, then we have

$$
\psi(u ; h) \leq \psi(u ; h-\widetilde{h}) \exp \left(-\frac{1}{2}\left\|\widetilde{h}_{1}\right\|^{2}\left\|\widetilde{h}_{2}\right\|^{2} \prod_{i=1,2} \int_{[0,1]} u_{i}(s) d\left(-\widetilde{h}_{i}^{\prime}(s)\right)\right)
$$

with $h:=h_{1} \times h_{2}, \tilde{h}:=\widetilde{h}_{1} \times \widetilde{h}_{2}, u:=u_{1} \times u_{2}$, and further

$$
\begin{aligned}
\psi(u ; h) \geq & \mathbf{P}\left\{l_{1}(s) l_{2}(t) \leq B(s, t) \leq u_{1}(s) u_{2}(t), \forall s, t \in[0,1]\right\} \\
& \times \exp \left(-\frac{1}{2}\left\|\widetilde{h}_{1}\right\|^{2}\left\|\widetilde{h}_{2}\right\|^{2}+\prod_{i=1,2} \int_{[0,1]} l_{i}(s) d\left(-\widetilde{h}_{i}^{\prime}(s)\right)\right) .
\end{aligned}
$$


Corollary 4.3 Under the assumptions and the notation of Corollary 4.2, if further $\min _{s \in[0,1]} u_{i}(s)>C \in(0, \infty), i=1,2$, and $u_{i}, i=1,2$, are absolutely continuous with $u_{i}^{\prime}$ satisfying $\int_{[0,1]}\left(u_{i}^{\prime}(s)\right)^{2} \lambda(d s)<\infty$, then we have

$$
\begin{aligned}
& \psi\left(u_{1} \times u_{2} ; \gamma h_{1} \times h_{2}\right) \\
& \quad=\exp \left(-\frac{\gamma^{2}}{2}\left\|\widetilde{h}_{1}\right\|^{2}\left\|\widetilde{h}_{2}\right\|^{2}+\gamma \prod_{i=1}^{2} \int_{[0,1]} u_{i}(s) d\left(-\widetilde{h}_{i}^{\prime}(s)\right)+z(\gamma)\right)
\end{aligned}
$$

with $z(\gamma)$ satisfying

$$
\begin{aligned}
-A \gamma^{2 / 3} \ln ^{3} \gamma & \leq z(\gamma) \\
& \leq \ln \mathbf{P}\left\{B_{0}(s, t) \leq u_{1}(s) u_{2}(t), \forall s, t \in[0,1]: \widetilde{h}_{1}(s) \widetilde{h}_{2}(t)=h_{1}(s) h_{2}(t)\right\}
\end{aligned}
$$

for all large $\gamma$, where $A$ is a positive constant not depending on $\gamma$. Furthermore

$$
\psi(u ; 0) \leq \inf _{h_{1}, h_{2} \in \mathcal{H}_{1}^{0}:\left\|\widetilde{h}_{1}\right\|\left\|\widetilde{h}_{2}\right\|>0} \Phi\left(\left(\left\|\widetilde{h}_{1}\right\|\left\|\widetilde{h}_{2}\right\|\right)^{-1} \prod_{i=1,2} \int_{[0,1]} u_{i}(s) d\left(-\widetilde{h}_{i}^{\prime}(s)\right)\right) .
$$

\section{Proofs}

Proof of Lemma 2.1 Let $g, h \in \mathcal{H}_{2}^{0}$ be two given functions. If $h^{\prime \prime} \in B V_{H}\left([0,1]^{2}\right)$ with $h^{\prime \prime}$ a right-continuous partial derivative of $h$, then we have by (6.2) and the integration by parts formula (see Lemmas 2 and 3 in [25] and (6.1))

$$
\begin{aligned}
\langle g, h\rangle & =\int_{[0,1]^{2}} g^{\prime \prime}(s, t) h^{\prime \prime}(s, t) \lambda^{2}(d s, d t) \\
& =\int_{[0,1]^{2}} h^{\prime \prime}(s, t) d g(s, t) \\
& =\int_{[0,1]^{2}} g(s, t) d h^{\prime \prime}(s, t) .
\end{aligned}
$$

Consequently, for any $g \in V$, by the assumption on $\widetilde{V}_{p, h}^{\prime \prime}$ we have $\left\langle g, \widetilde{V}_{p, h}\right\rangle \leq 0$. Hence for any function $g:[0,1]^{2} \rightarrow[0, \infty)$ which is Riemann-Stieltjes integrable with respect to $\widetilde{V}_{p, h}^{\prime \prime}$ on $[0,1]^{2}$, for the corresponding Riemann-Stieltjes integral, we have

$$
\int_{[0,1]^{2}} g(s, t) d \widetilde{V}_{p, h}^{\prime \prime} \geq 0 .
$$

The proof of statements (b) and (c) follows immediately by Lemma 2 in [15]. 
We show next statement (d). Let $\tilde{h} \in \mathcal{H}_{2}^{0}$ be a given function such that $\tilde{h}:=g+h$ with $g(s, t) \geq 0, \forall s, t \in[0,1]$. By the properties of $\widetilde{V}_{p, h}$ we have $\left\langle\widetilde{V}_{p, h}, g\right\rangle \geq 0$, hence we may write

$$
\begin{aligned}
\|\widetilde{h}\|^{2} & =\|g+h\|^{2} \\
& =\left\|\widetilde{V}_{p, h}+g+h-\widetilde{V}_{p, h}\right\|^{2} \\
& =\left\|\widetilde{V}_{p, h}\right\|^{2}+2\left\langle\widetilde{V}_{p, h}, g+h-\widetilde{V}_{p, h}\right\rangle+\left\|g+h-\widetilde{V}_{p, h}\right\|^{2} \\
& =\left\|\widetilde{V}_{p, h}\right\|^{2}+2\left\langle\widetilde{V}_{p, h}, g\right\rangle+2\left\langle V_{p, h}, \widetilde{V}_{p, h}\right\rangle+\left\|g+h-\widetilde{V}_{p, h}\right\|^{2} \\
& =\left\|\widetilde{V}_{p, h}\right\|^{2}+2\left\langle\widetilde{V}_{p, h}, g\right\rangle+\left\|g+h-\widetilde{V}_{p, h}\right\|^{2} \\
& \geq\left\|\widetilde{V}_{p, h}\right\|^{2}+2\left\langle\widetilde{V}_{p, h}, g\right\rangle \\
& \geq\left\|\widetilde{V}_{p, h}\right\|^{2} .
\end{aligned}
$$

Since further $\widetilde{V}_{p, h}(s, t) \geq h(s, t), \forall s, t \in[0,1]$, it follows that the solution of the minimisation problem (2.2) is $\widetilde{V}_{p, h}$. Clearly, its solution is unique, and thus the result follows.

Proof of Proposition 3.1 By (2.3) and (3.2) we see that (3.4) follows easily. The proof of (3.5) can be established along the lines of the proof of Lemma 5 in [15], thus the result.

Proof of Proposition 3.2 Let $V, \widetilde{V}$ be as in Section 2, and let $\widetilde{V}_{p, h}$ be the projection of $h$ into the polar cone $\widetilde{V}$. In view of statement (b) of Lemma 2.1,

$$
h=V_{p, h}+\widetilde{V}_{p, h}, \quad\|h\|^{2}=\left\|\widetilde{V}_{p, h}\right\|^{2}+\left\|V_{p, h}\right\|^{2} .
$$

Furthermore, $\psi(u ; h) \geq \psi\left(u ; \widetilde{V}_{p, h}\right)$. Next, applying the Cameron-Martin formula, we obtain (set $\mathbf{1}_{u}\left(B_{0}(s, t)\right):=\mathbf{1}\left(B_{0}(s, t) \leq u(s, t), \forall s, t \in[0,1]\right)$

$$
\begin{aligned}
\psi(u ; h)= & \exp \left(-\frac{1}{2}\|h\|^{2}\right) \mathbf{E}\left\{\exp \left(\int_{[0,1]^{2}} h^{\prime \prime}(s, t) d B_{0}(s, t)\right) \mathbf{1}_{u}\left(B_{0}(s, t)\right)\right\} \\
= & \exp \left(-\frac{1}{2}\left\|\widetilde{V}_{p, h}\right\|^{2}\right) \mathbf{E}\left\{\operatorname { e x p } \left(-\frac{1}{2}\left\|V_{p, h}\right\|^{2}+\int_{[0,1]^{2}} V_{p, h}^{\prime \prime}(s, t) d B_{0}(s, t)\right.\right. \\
& \left.\left.+\int_{[0,1]^{2}} \widetilde{V}_{p, h}^{\prime \prime}(s, t) d B_{0}(s, t)\right) \mathbf{1}_{u}\left(B_{0}(s, t)\right)\right\} .
\end{aligned}
$$

Since $\widetilde{V}_{p, h}^{\prime \prime} \in B V_{H}\left([0,1]^{2}\right)$ is right continuous and $B_{0}(s, t)$ has continuous sample paths, by the integration by parts formula (6.1) for the Riemann-Stieltjes integral we have almost surely

$$
\int_{[0,1]^{2}} B_{0}(s, t) d \widetilde{V}_{p, h}^{\prime \prime}(s, t)=\int_{[0,1]^{2}} \widetilde{V}_{p, h}^{\prime \prime}(s, t) d B_{0}(s, t) .
$$


Consequently, we may further write (recall (5.2))

$$
\begin{aligned}
\psi(u ; h)= & \mathbf{E}\left\{\operatorname { e x p } \left(-\frac{1}{2}\left\|V_{p, h}\right\|^{2}+\int_{[0,1]^{2}} V_{p, h}^{\prime \prime}(s, t) d B_{0}(s, t)\right.\right. \\
& \left.\left.+\int_{[0,1]^{2}} B_{0}(s, t) d \widetilde{V}_{p, h}^{\prime \prime}(s, t)\right) \mathbf{1}_{u}\left(B_{0}(s, t)\right)\right\} \\
\leq & \exp \left(-\frac{1}{2}\left\|\widetilde{V}_{p, h}\right\|^{2}+\int_{[0,1]^{2}} u(s, t) d \widetilde{V}_{p, h}^{\prime \prime}(s, t)\right) \\
& \times \mathbf{E}\left\{\exp \left(-\frac{1}{2}\left\|V_{p, h}\right\|^{2}+\int_{[0,1]^{2}} V_{p, h}^{\prime \prime}(s, t) d B_{0}(s, t)\right) \mathbf{1}_{u}\left(B_{0}(s, t)\right)\right\} \\
= & \exp \left(-\frac{1}{2}\left\|\widetilde{V}_{p, h}\right\|^{2}+\int_{[0,1]^{2}} u(s, t) d \widetilde{V}_{p, h}^{\prime \prime}(s, t)\right) \psi\left(u ; V_{p, h}\right) .
\end{aligned}
$$

Clearly, by the definition $\psi(u ; h) \geq \psi\left(u ; \widetilde{V}_{p, h}\right)$. Applying (3.7) to $\psi\left(u ; \gamma \widetilde{V}_{p, h}\right)$, $\gamma>0$, we find

$$
\ln \psi(u ; \gamma h)=-(1+o(1)) \frac{\gamma^{2}}{2}\left\|\widetilde{V}_{p, h}\right\|^{2}, \quad \gamma \rightarrow \infty
$$

hence by (3.7) the unique solution of (2.2) equals $\widetilde{V}_{p, h}$. Since $\widetilde{V}_{p, h} \geq h$ and $\widetilde{V}_{p, h} \in \widetilde{V}$, we have $\underline{h}=\widetilde{V}_{p, h}$, and (3.8) follows.

We next show the last claim (3.10). Using again the Cameron-Martin formula, we have

$$
\begin{aligned}
\psi(u ; h) \geq & \psi(u ; \underline{h}) \\
\geq & \mathbf{P}\left\{l(s, t) \leq B_{0}(s, t)+\underline{h}(s, t) \leq u(s, t), \forall s, t \in[0,1]\right\} \\
= & \exp \left(-\frac{1}{2}\|\underline{h}\|^{2}\right) \mathbf{E}\left\{\exp \left(\int_{[0,1]^{2}} \underline{h}^{\prime \prime}(s, t) d B_{0}(s, t)\right)\right. \\
& \left.\times \mathbf{1}\left(l(s, t) \leq B_{0}(s, t) \leq u(s, t), \forall s, t \in[0,1]\right)\right\} \\
= & \mathbf{P}\left\{l(s, t) \leq B_{0}(s, t) \leq u(s, t), \forall s, t \in[0,1]\right\} \\
& \times \exp \left(-\frac{1}{2}\|\underline{h}\|^{2}+\int_{[0,1]^{2}} l(s, t) d \underline{h}^{\prime \prime}(s, t)\right),
\end{aligned}
$$

hence the proof is established.

\section{Proof of Proposition 3.3 Set next}

$$
\underline{h}_{\epsilon}(s, t):=\underline{h}(s, t)-u_{\epsilon}(s, t), \quad \forall s, t \in[0,1] .
$$


Applying the Cameron-Martin formula, we obtain

$$
\begin{aligned}
\psi(u ; h) \geq & \psi(u ; \underline{h}) \\
= & \mathbf{P}\left\{B_{0}(s, t)+\underline{h}(s, t) \leq u(s, t), \forall s, t \in[0,1]\right\} \\
\geq & \mathbf{P}\left\{B_{0}(s, t)+\underline{h}(s, t) \leq u_{\epsilon}(s, t)+\epsilon, \forall s, t \in[0,1]\right\} \\
> & \exp \left(-\frac{1}{2}\|\underline{h} \epsilon\|^{2}\right) \mathbf{E}\left\{\exp \left(\int_{[0,1]^{2}} \underline{h}_{\epsilon}^{\prime \prime}(s, t) d B_{0}(s, t)\right)\right. \\
& \left.\times \mathbf{1}\left(-\epsilon \leq B_{0}(s, t) \leq \epsilon, \forall s, t \in[0,1]\right)\right\} .
\end{aligned}
$$

Define the Gaussian random variable

$$
Z:=\int_{[0,1]^{2}} \underline{h}_{\epsilon}^{\prime \prime}(s, t) d B_{0}(s, t) .
$$

Clearly, $Z$ has mean 0 and variance $\left\|\underline{h}_{\epsilon}\right\|^{2}$. For $\varepsilon>0$ small enough, we have $\left\|\underline{h}_{\epsilon}\right\| \epsilon$ $(0, \infty)$. For any constant $C \in \mathbb{R}$ and $\varepsilon$ small enough, we may write

$$
\begin{aligned}
& \mathbf{E}\left\{\exp (Z) \mathbf{1}\left(-\epsilon \leq B_{0}(s, t) \leq \epsilon, \forall s, t \in[0,1]\right)\right\} \\
&=\mathbf{E}\left\{\exp (Z) \mathbf{1}\left(-\epsilon \leq B_{0}(s, t) \leq \epsilon, \forall s, t \in[0,1]\right)[\mathbf{1}(Z<C)+\mathbf{1}(Z \geq C)]\right\} \\
& \geq \mathbf{E}\left\{\exp (Z) \mathbf{1}\left(-\epsilon \leq B_{0}(s, t) \leq \epsilon, \forall s, t \in[0,1]\right) \mathbf{1}(Z \geq C)\right\} \\
& \geq \exp (C) \mathbf{P}\left\{-\epsilon \leq B_{0}(s, t) \leq \epsilon, \forall s, t \in[0,1], Z \geq C\right\} \\
&=\exp (C)\left[\mathbf{P}\left\{\sup _{s, t \in[0,1]}\left|B_{0}(s, t)\right|<\epsilon\right\}\right. \\
&\left.-\mathbf{P}\left\{-\epsilon \leq B_{0}(s, t) \leq \epsilon, \forall s, t \in[0,1], Z<C\right\}\right] \\
& \geq \exp (C)\left[\mathbf{P}\left\{\sup _{s, t \in[0,1]}\left|B_{0}(s, t)\right|<\epsilon\right\}-\mathbf{P}\{Z \leq C\}\right] \\
&= \exp (C)\left[\mathbf{P}\left\{\sup _{s, t \in[0,1]}\left|B_{0}(s, t)\right|<\epsilon\right\}-\Phi(C /\|\underline{h} \epsilon\|)\right] .
\end{aligned}
$$

By the small ball asymptotic result (see $[7-9,16])$ we have

$$
\boldsymbol{P}\left\{\sup _{s, t \in[0,1]}\left|B_{0}(s, t)\right|<\epsilon\right\} \geq \exp \left(-K \frac{\ln ^{3}(1 / \epsilon)}{\epsilon^{2}}\right)
$$

for some positive constant $K$ and all $\epsilon>0$ small enough. Since

$$
\left\|\underline{h}_{\epsilon}\right\|^{2}=\|\underline{h}\|^{2}-2 \int_{[0,1]^{2}} u_{\varepsilon}(s, t) d \underline{h}^{\prime \prime}(s, t)+\left\|u_{\varepsilon}\right\|^{2}=O\left(1 / \varepsilon^{2}\right),
$$

choosing $C:=-K_{*}\left\|\underline{h}_{\epsilon}\right\| \ln ^{3 / 2}(1 / \epsilon) / \epsilon, K_{*} \in(0, \infty), K_{*}^{2}>K$ and using the Millsratio asymptotics for Gaussian random variables for all $\epsilon>0$ small enough and some 
positive constants $c_{1}, c_{2}$, we have

$$
\mathbf{E}\left\{\exp (Z) \mathbf{1}\left(-\epsilon \leq B_{0}(s, t) \leq \epsilon, \forall s, t \in[0,1]\right)\right\} \geq \exp \left(-\frac{c_{1}}{\epsilon}-\frac{c_{2} \ln ^{3}(1 / \epsilon)}{\epsilon^{2}}\right)
$$

implying thus

$$
\psi(u ; h) \geq \exp \left(-\frac{1}{2}\|\underline{h}\|^{2}+\int_{[0,1]^{2}} u_{\epsilon}(s, t) d \underline{h}^{\prime \prime}(s, t)-\frac{c_{1}}{\epsilon}-\frac{c_{2} \ln ^{3}(1 / \epsilon)}{\epsilon^{2}}\right) .
$$

Recalling that $\lim _{\varepsilon \rightarrow 0} u_{\epsilon}(s, t)=u(s, t), \forall s, t \in[0,1]$ and $\left\|u_{\epsilon}\right\|^{2}=O\left(1 / \varepsilon^{2}\right)$, we obtain using the result of Proposition 3.2 (set next $\epsilon:=\gamma^{-1 / 3}, \gamma>0$ )

$$
\psi(u ; \gamma h)=\exp \left(-\frac{\gamma^{2}}{2}\|\underline{h}\|^{2}+\gamma I+z(\gamma)\right), \quad \gamma \rightarrow \infty,
$$

where $|I| \leq M$ with $I:=\int_{[0,1]^{2}} u(s, t) d \underline{h}^{\prime \prime}(s, t)$ and

$$
-A \gamma^{2 / 3} \ln ^{3} \gamma \leq z(\gamma) \leq \ln \boldsymbol{P}\left\{B_{0}(s, t) \leq u(s, t), \forall s, t \in[0,1]: \underline{h}(s, t)=h(s, t)\right\}
$$

is satisfied for all $\gamma$ large and a positive constant $A$ not depending on $\gamma$. Hence the result follows.

Proof of Lemma 4.1 Set $V:=\left\{h \in \mathcal{H}_{2}^{0}: h(s, t) \leq 0, \forall s, t \in[0,1]\right\}$ and $\underline{h}:=\widetilde{h}_{1} \times \widetilde{h}_{2}$. By the assumptions the function $g:=\underline{h}-h_{1} \times h_{2}$ belongs to $V$. Furthermore, for any $v \in V$, we have

$$
\langle v, \underline{h}\rangle=\int_{[0,1]^{2}} v(s, t) d\left(\widetilde{h}_{1}^{\prime}(s) \widetilde{h}_{2}^{\prime}(t)\right) \leq 0 .
$$

Consequently $\underline{h}$ belongs to the polar cone $\widetilde{V}$ of $V$. In view of statement (c) in Lemma 2.1, the proof follows if we show that $g$ is orthogonal to $\underline{h}$. Since $\widetilde{h}_{i}-h_{i}$ is orthogonal to $\widetilde{h}_{i}, i=1,2$ (see [2]), we have

$$
\begin{aligned}
\langle g, \underline{h}\rangle & =\left\langle\widetilde{h}_{1} \times \widetilde{h}_{2}-h_{1} \times h_{2}, \tilde{h}_{1} \times \widetilde{h}_{2}\right\rangle \\
& =\left\langle\widetilde{h}_{1} \times\left(\widetilde{h}_{2}-h_{2}\right), \widetilde{h}_{1} \times \widetilde{h}_{2}\right\rangle-\left\langle\left(\widetilde{h}_{1}-h_{1}\right) \times h_{2}, \widetilde{h}_{1} \times \widetilde{h}_{2}\right\rangle \\
& =0,
\end{aligned}
$$

hence the result follows.

Proof of Corollary 4.3 The proof follows easily by the assumptions on $u_{i}, i=1,2$.

Acknowledgements I would like to thank a Referee and Professor Wembo Li for several corrections and suggestions, Professor Móricz for sending [25] and Professors Muhammad Aslam Noor and Wolfgang Bischoff for some insights on Hilbert spaces. 


\section{Appendix}

In this short section we provide two results for the Riemann-Stieltjes integral.

Let $f:[0,1]^{2} \rightarrow \mathbb{R}$ be a given function. If $f(s, t)=g(s, t)+g_{1}(s)+g_{2}(t)$ with $g \in B V_{H}\left([0,1]^{2}\right)$ and $g_{1}, g_{2}$ two other functions, then $h$ has bounded variation in the sense of Vitali (write $f \in B V_{V}\left([0,1]^{2}\right)$ ). In fact $f$ can be expressed as the difference of two real functions defined on $[0,1]^{2}$ which generate a positive measure on $[0,1]^{2}$. Thus the class of functions with bounded variation in the sense of Vitali consists of all real functions defined on $[0,1]^{2}$ generating a finite signed measure.

If $g:[0,1]^{2} \rightarrow \mathbb{R}$ is continuous, then it is well known that the Riemann-Stieltjes integral $\int_{[0,1]^{2}} g(x, y) d f(x, y)$ exists, provided that $f \in B V_{V}\left([0,1]^{2}\right)$. In the next lemma we present an integration by parts formula; the case $f \in B V_{H}\left([0,1]^{2}\right)$ is discussed in Lemma 1 in [25].

Lemma 6.1 Let $f, g:[0,1]^{2} \rightarrow \mathbb{R}$ be two given functions. If $g$ is continuous such that $g(s, t)=0$ for all $(s, t)$ in the boundary of $[0,1]^{2}$ and $f \in B V_{V}\left([0,1]^{2}\right)$, then the integration by parts formula for the Riemann-Stieltjes integral reads

$$
\int_{[0,1]^{2}} g(x, y) d f(x, y)=\int_{[0,1]^{2}} f(x, y) d g(x, y) .
$$

Proof The proof follows with similar arguments as in Lemma 2 in [25], since the four single sums in expression (3.8) therein are equal to 0 due to the fact that $g$ vanishes on the boundary of $[0,1]^{2}$.

Lemma 6.2 Let $f, g:[0,1]^{2} \rightarrow \mathbb{R}$ be two given functions. Assume that $g$ is absolutely continuous with $g(s, t)=\int_{[0, s] \times[0, t]} h(x, y) \lambda^{2}(d x, d y), s, t \in[0,1]$. If $f \in B V_{V}\left([0,1]^{2}\right)$ and $f$ is almost surely continuous with respect to $\lambda^{2}$, then we have

$$
\int_{[0,1]^{2}} g(x, y) d f(x, y)=\int_{[0,1]^{2}} f(x, y) h(x, y) d \lambda^{2}(d x, d y) .
$$

Proof The proof follows with similar arguments as in Lemma 3 in [25].

\section{References}

1. Adams, C.R., Clarkson, J.A.: Properties of functions $f(x, y)$ of bounded variation. Trans. Am. Math. Soc. 36, 711-730 (1934)

2. Bischoff, W., Hashorva, E.: A lower bound for boundary crossing probabilities of Brownian bridge/motion with trend. Stat. Probab. Lett. 74(3), 265-271 (2005)

3. Bischoff, W., Miller, F., Hashorva, E., Hüsler, J.: Asymptotics of a boundary crossing probability of a Brownian bridge with general trend. Methodol. Comput. Appl. Probab. 5(3), 271-287 (2003)

4. Bischoff, W., Hashorva, E., Hüsler, J., Miller, F.: Analysis of a change-point regression problem in quality control by partial sums processes and Kolmogorov type tests. Metrika 62, 85-98 (2005)

5. Borovkov, K., Novikov, A.: Explicit bounds for approximation rates of boundary crossing probabilities for the Wiener process. J. Appl. Probab. 42, 82-92 (2005)

6. Csáki, E., Khoshnevisan, D., Shi, Z.: Boundary crossings and the distribution function of the maximum of Brownian sheet. Stoch. Process. Appl. 90, 1-18 (2000) 
7. Fill, J., Torcaso, F.: Asymptotic analysis via Mellin transforms for small deviations in $L_{2}$-norm of integrated Brownian sheets. Probab. Theory Relat. Fields 130, 259-288 (2004)

8. Gao, F., Li, W.V.: Logarithmic level comparison for small deviation probabilities. J. Theor. Probab. 19(3), 535-556 (2006)

9. Gao, F., Li, W.V.: Small ball probabilities for the Slepian Gaussian fields. Trans. Am. Math. Soc. 359, 1339-1350 (2007)

10. Gao, F., Hannig, J., Lee, T.-Y., Torcaso, F.: Exact $L_{2}$ small balls of Gaussian processes. J. Theor. Probab. 17(2), 503-520 (2004)

11. Goovaerts, M.J., Teunen, M.: Boundary Crossing Results for the Brownian motion. Blätter (1993), pp. 197-205

12. Hashorva, E.: Exact asymptotics for boundary crossing probabilities of Brownian motion with piecewise linear trend. Electron. Commun. Probab. 10, 207-217 (2005)

13. Hashorva, E.: Asymptotics and bounds for multivariate Gaussian tails. J. Theor. Probab. 18(1), 79-97 (2005)

14. Janssen, A., Kunz, M.: Boundary crossing probabilities for piece-wise linear boundary functions. Commun. Stat. Theory Methods 33(7), 1445-1464 (2004)

15. Janssen, A., Ünlü, H.: Regions of alternatives with high and low power for goodness-of-fit tests. J. Stat. Plan. Inference 138, 2526-2543 (2008)

16. Karol', A., Nazarov, A., Nikitin, Y.: Small ball probabilities for Gaussian random fields and tensor products of compact operators. Trans. Am. Math. Soc. 360, 1443-1474 (2008)

17. Koning, A.J., Protasov, V.: Tail behaviour of Gaussian processes with applications to the Brownian pillow. J. Multivar. Anal. 82(2), 370-397 (2003)

18. Khoshnevisan, D., Pemantle, R.: Sojourn times of Brownian sheet. Period. Math. Hung. 41(1-2), 187-194 (2000)

19. Kuelbs, J.: A strong convergence theorem for Banach space valued random variables. Ann. Probab. 4, 744-771 (1976)

20. Kuelbs, J., Li, W.V.: Small ball estimates for Brownian motion and the Brownian sheet. J. Theor. Probab. 6(3), 547-577 (1992)

21. Ledoux, M.: Isoperimetry and Gaussian Analysis. Lectures on Probability Theory and Statistics. Lecture Notes in Math., vol. 1648. Springer, Berlin (1996)

22. Li, W.V., Kuelbs, J.: Some shift inequalities for Gaussian measures. Prog. Probab. 43, 233-243 (1998)

23. Li, W.V., Shao, Q.M.: Gaussian processes: Inequalities, small ball probabilities and applications. In: Rao, C.R., Shanbhag, D. (eds.) Stochastic Processes: Theory and Methods Handbook of Statistics, vol. 19, pp. 533-597. North-Holland, Amsterdam (2001)

24. Lifshits, M.A.: Gaussian Random Functions. Mathematics and Its Applications, vol. 322, Kluwer Academic, Dordrecht (1995)

25. Móricz, F.: Order of magnitude of double Fourier coefficients of functions of bounded variation. Analysis (Munich) 22, 335-345 (2002)

26. Novikov, A.A., Frishling, V., Kordzakhia, N.: Approximations of boundary crossing probabilities for a Brownian motion. J. Appl. Probab. 36, 1019-1030 (1999)

27. Pötzelberger, K., Wang, L.: Boundary crossing probability for Brownian motion. J. Appl. Probab. 38, 152-164 (2001)

28. van der Vaart, A.W., Wellner, J.A.: Weak Convergence and Empirical Processes, with Applications to Statistics. Springer, New York (1996)

29. Wang, L., Pötzelberger, K.: Boundary crossing probability for Brownian motion and general boundaries. J. Appl. Probab. 34, 54-65 (1997) 